\title{
Impact of Exogenous Neurotropic Factor Administration on Occurrence and Severity of Paclitaxel-Induced Neuropathy in Breast Cancer Patients: An Open Label Pilot Study
}

\author{
Mahmoud A. Ellithy ${ }^{1}$, Lamia Elwakil ${ }^{2}$, Mona Schaalan ${ }^{3}$, Yomna A. El Hossamy ${ }^{3}$ \\ ${ }^{1}$ Clinical Oncology Department, Faculty of Medicine, Ain Shams University, Cairo, Egypt; ${ }^{2}$ Faculty \\ of Pharmacy, Ain Shams University, Cairo, Egypt; ${ }^{3}$ Clinical Pharmacy Department, Faculty of \\ Pharmacy, Misr International University, Cairo, Egypt
}

Background: Paclitaxel-induced peripheral neuropathy (PIPN) is a common toxicity with no proven agent beneficial for prevention. The potentiality of Nerve Growth Factor (NGF) as a protective agent for PIPN was suggested by several studies.

Aim: This study aimed to test the impact of exogenously administered NGF on PIPN and to assess NGF levels in relation to PIPN severity.

Methods: Forty patients were prospectively randomly allocated to paclitaxel alone (control group) or paclitaxel + exogenous NGF (test group). Neuropathy occurrence and severity was assessed before enrollment and after each cycle using the European Association for Treatment of Cancer Quality of Life Questionnaire for Chemotherapy Induced Peripheral Neuropathy (EORTC QLQ-CIPN20). Nerve Growth Factor level was assessed in both groups at baseline and at the end of the study. Nerve Growth Factor safety was assessed by laboratory investigations and the occurrence of adverse drug reactions.

Results: There was significant increase in the EORTC QLQ-CIPN20 score in the control group ( $p<0.001)$ and a stabilization in the score in the test group. Nerve Growth Factor levels significantly increased in the test group (p<0.001) and declined in the control group. A highly significant negative correlation existed between NGF level and the EORTC QLQ-CIPN20 score $(r=-0.781, p<0.001)$. No significant difference was observed between the two groups in the occurrence of adverse drug reactions or other toxicities.

Conclusion: Exogenous NGF may have a potential neuroprotective effect against PIPN in breast cancer patients. Higher endogenous NGF level is inversely correlated with the occurrence and severity of PIPN.

Keywords: Nerve Growth Factor, Neuropathy, Paclitaxel, Breast cancer.

Corresponding author: Mahmoud A. Ellithy, MD; Clinical Oncology Department, Faculty of Medicine, Ain Shams University, Cairo, Egypt ; Email: ellithym@med.asu.edu.eg

Submitted: 30-March-2018, Revised: 25-April-2018, Accepted: 30-April-2018, Published online: 29-June-2018

\section{INTRODUCTION}

Chemotherapy-induced toxicities are challenging problems for cancer patients. These toxicities not only impair patients' quality of life but may also lead to treatment dose reduction or discontinuation. Neuropathic changes induced by chemotherapy is a common adverse effect of many anticancer drugs ${ }^{1}$.

Paclitaxel is a cytotoxic agent that exhibits high antitumor activity through inhibition of microtubules during the cell division ${ }^{2}$. Cumulative peripheral neuropathy and rapid-onset sensory neuropathy are common toxicities that may limit the use of paclitaxel ${ }^{3}$. Paclitaxel-induced peripheral neuropathy (PIPN) is due to axonal degeneration of the sensory afferents of the epidermis. Motor and autonomic nerves appear to be unaffected by paclitaxel ${ }^{4}$.

Taxane-associated peripheral neuropathy can be classified into either motor or sensory, according to the type of nerve fibers involved 5 . Breast cancer patients treated with taxane-based adjuvant chemotherapy may develop neuropathy symptoms up to two years after therapy completion ${ }^{6}$. Multiple mechanisms have been implicated in PIPN, inhibition of microtubule function, impairment of mitochondrial function and evoke spontaneous discharges from sensory neurons resulting in clinically evident PIPN ${ }^{7}$. Neuronal damage has also been proven to be related to the frequency and duration of paclitaxel infusion ${ }^{8}$.

Primary afferent nociceptive fibers are destroyed by taxanes at its terminal arbors inside the epidermis 9,10 . Survival of neurons, growth and maintenance is regulated with the nerve growth factor (NGF) $11,12$. There is positive correlation between the occurrence of neuropathy and reduced NGF level ${ }^{13}$. Studies showed that patients with neuropathy induced by chemotherapy have reduced serum levels of endogenous NGF ${ }^{14}$.

Objective assessment of neuropathy can be invasive or non invasive ${ }^{1}$. Nerve conduction studies are invasive methods ${ }^{15}$. Subjective assessment includes the use of the National Cancer Institute Common Terminology Criteria for Adverse Events (NCI-CTCAE) ${ }^{16}$. Another tool to 
assess chemotherapy-induced neuropathy is the European Association for Treatment of Cancer Quality of Life Questionnaire for Chemotherapy-Induced Peripheral Neuropathy (EORTC QLQ-CIPN20) ${ }^{17}$. The EORTC QLQ-CIPN20 module evaluates sensory, motor and autonomic neuropathies ${ }^{18}$.

Exogenous NGF (cerebrolysin) is formed from multiple neuropeptides in addition to amino acids obtained from the brain of young porcine. The neuroprotective action of cerebrolysin is achieved by inhibiting free radicals and reducing lipid peroxides in addition to inhibiting glutamate excitotoxicity and preventing neuronal death due to hypoxia and ischemia.. It has a nerve growth factor activity through the stimulation of neuronal differentiation and sprouting ${ }^{19}$.

The current study was designed to evaluate the impact of cerebrolysin administration on the occurrence and severity of PIPN.

\section{METHODS}

The study was an open-label, randomized, controlled pilot study. It was conducted at the Clinical Oncology Department of Ain Shams University Hospital in cooperation with the Clinical Pharmacy Department, Faculty of Pharmacy, Ain Shams University and the Faculty of Pharmacy, Misr International University.

An informed signed consent was obtained from all participants and the study was approved by the Research Ethics Committee of the Clinical Oncology Department, Faculty of Medicine, Ain Shams University dated November 2014 and by the Ethical Research Committee of the Faculty of Pharmacy, Ain Shams University. The study registration number in the Cure and More Egyptian National Research Consortium is RWN005. All procedures performed in studies followed the rules and the ethical standards of the Institutional Research Committee and with the 1964 Helsinki declaration and its later modifications..

\section{Patient recruitment}

All patients admitted to the oncology department from January 2015 to October 2016 were assessed for eligibility prior to inclusion in the study.

Inclusion criteria: newly diagnosed male or female breast cancer patients with an age $\geq 18$ and $\leq 60$ years, treatment with 3 -weekly paclitaxel monotherapy (135$175 \mathrm{mg} / \mathrm{m}^{2}$ administered over three hours) for the first time and acceptance to take cerebrolysin intramuscular injection according to the designed schedule of the study. The investigators intended to use 3-weekly paclitaxel rather than the weekly schedule because many patients are living in far districts away from the hospital and may not be compliant with the weekly schedule. In Egypt, a patient can take intramuscular injection at any pharmacy as allowed by the Egyptian Ministry of Health.

Exclusion criteria; non-compliance to anticancer medications, previous exposure to chemotherapy drugs known to cause neuropathy, treatment with medications that increase the risk of peripheral neuropathy (amiodarone, chloramphenicol, chloroquine, colchicines, dapsone, fluoroquinolones, hydralazine, isoniazide, metronidazole, nitrofurantoi, phenytoin, procainamide and statins), comorbid conditions with risk factors for neuropathies (diabetes mellitus, alcoholism and paraneoplastic and dysmetabolic syndromes), personal history of peripheral neuropathy, impaired renal function (creatinine clearance $<60 \mathrm{ml} / \mathrm{min}$ ), impaired liver function (liver enzymes $>2.5$ folds of high normal), epilepsy, vitamin B replacement therapy, illiteracy, cognitive impairment, psychological instability and vision problems that may impair ability to read and answer the questionnaire.

Prior to enrollment, all patients were assessed by three experts, clinical pharmacist, clinical oncologist and neurologist for eligibility. The clinical pharmacist assessed the drug history of the patients, the clinical oncologist assessed the disease condition (tumor staging) and the neurologist did base line clinical neurological examination for all cases. All investigators received training on QLQ-CIPN20 administration.

\section{Treatment}

Forty eligible patients were randomly assigned through a one to one randomization to either the control group ( 3 cycles of paclitaxel without cerebrolysin) or the test group (3 cycles of paclitaxel + cerebrolysin). Cerebrolysin treatment was administered as a $5 \mathrm{ml}$ intramuscular injection once/day starting on the day before paclitaxel administration, then every other day for one week, then discontinued till the next paclitaxel cycle. The exogenous NF (cerebrolysin) used in the study was manufactured by Ebwe Pharma ${ }^{20,21}$.

\section{Assessment}

The occurrence of neuropathy and its severity was assessed using a self-administered Arabic translation of the QLQ-CIPN20 ${ }^{18}$. The investigators got the permission of the EORTC to translate the QLQ-CIPN20 to Standard Arabic for the purpose of this study. The QLQ-CIPN20 contains questions assessing sensory, motor and autonomic neuropathy. Nine, 8 and 3 questions were used to assess sensory, motor and autonomic manifestations respectively. Respondents answer the questions on a 4-point Likert scale (1 = "not at all," 2 = "a little," 3 = "quite a bit," and 4 = "very much"). The answers indicate to which degree the patient had experienced sensory, motor, and autonomic symptoms during the past week. Autonomic scale range from 3 to 12 for men and 2-8 for women (erectile function item is excluded). Sensory raw score ranges from 9 to 36 and motor raw score range from 8 to 32 . Total score is calculated by adding the sensory, motor and autonomic scores. The results are linearly converted to a 0-100 scale, with higher scores indicating more symptom burden as recommended in Postma et al study that was cited in EORTC manual ${ }^{18}$.

Patients were evaluated immediately before paclitaxel administration on days 1, 21 and 42 and on day 63 for QLQ-CIPN20 neuroscores and endogenous NGF levels.

The Enzyme- Linked Immunosorbent Assay (ELISA) kit CSB-E04683h (a commercial kit 
manufactured by Cusabio Biotech Company, Wuhan, Hubei Province, P. R. China) was used for measuring the endogenous human NGF. Blood samples were collected using EDTA tubes and were centrifuged at $1000 \mathrm{xg}$ for 15 minutes 30 minutes from the time of sample collection. The plasma was stored at $-20^{\circ} \mathrm{C}$ till the analysis time. The detection range of the kit was $78 \mathrm{pg} / \mathrm{ml}-5000 \mathrm{pg} / \mathrm{ml}$. The assay has high specificity and sensitivity for the detection of human NGF. No significant cross reactivity or interference between human NGF and analogues was observed.

The safety and tolerability of cerebrolysin was assessed by full laboratory assessment in addition to myalgia and arthralgia assessment at baseline and at the end of the study.

\section{End Points}

The end points of the current study were the change in neuroscores within each group and between the two groups and the change in endogenous NGF serum levels after 3 paclitaxel cycles.

Other end points included recruitment rate, refusal rate, non-compliance rate, time and budget problems and potential human and data optimization problems,.

\section{Statistical Analysis}

The small sample size was justified by the fact that we were piloting the idea to test its feasibility. Statistical Package for Social Sciences (SPSS) version 23 was used to manage and analyze the data. Means and standard deviations or medians and ranges were used to summarize data, as appropriate. Categorical data was summarized as numbers and percentages. Numerical data was explored for normality using KolmogrovSmirnov and Shapiro-Wilk tests. Comparison between two groups was done by Mann-Whitney test. Change between baseline and end of study was tested using the Wilcoxon Rank Signed. Changes from baseline and all the cycles were tested using the Friedman's test. To measure the strength of association between the measurements, Spearman's correlation coefficients were calculated. All p-values were two-sided and p values < 0.05 were considered significant.

\section{RESULTS}

Out of the total 54 patients who received paclitaxel during the study period, only 48 were eligible. Out of the 48 eligible patients; 7 refused to join the study, and one patient was non-compliant after joining the study and was excluded. The remaining 40 patients were randomly assigned to the study. The contribution rate was $85.41 \%$, the refusal rate was $14.5 \%$ and the non-compliance rate was $2.4 \%$.

No significant difference was observed between both group in terms of age, paclitaxel dose or body surface area (table 1). All cases were females with breast cancer.

The QLQ-CIPN20 neuroscores and endogenous NGG levels in both groups are shown in table 2. At baseline, there was no significant difference in the QLQCIPN20 neuroscores between groups, while a highly significant difference in the scores between groups at each cycle was observed. For test group, the neuroscors of cycle 1 was significantly different from cycle 2 and 3 . For control group, the neuroscores of cycle 1 was significantly different from cycle 3 only.The neuroscore increased significantly in the control group as compared to the test group.

The NGF levels were comparable between groups at baseline, while a highly significant difference was observed in the NGF levels between groups at the third cycle. The test group showed a highly significant increase in the final serum NGF level relative to its baseline levels, while the control group showed a significant reduction in the final NGF level compared to its initial values. The percentage change in NGF values was significantly higher for the test group as compared to the control group.

The Spearman correlation test showed a highly significant correlation between NGF levels and QLQCIPN20 neuroscores at the $3^{\text {rd }}$ cycle $(r=-0.781, p<0.001)$.

The myalgia and arthralgia scores and laboratory investigations results are illustrated in tables 3 and 4 respectively. There was no significant difference in all evaluated laboratory parameters (complete blood picture, aspartate transaminase, alanine transaminase, total bilirubin, random blood glucose, blood urea nitrogen, serum creatinine) between the two groups neither at baseline nor at the end of the study. None of the patients in the test group complained of any adverse drug reactions related to cerebrolysin. Administrated cerebrolysin did not potentiate any of the paclitaxel induced side effects and patients in the test group did not experience any adverse drug reactions related to cerebrolysin.

\section{DISCUSSION}

The prevalence of PIPN in surveyed patients from January 2014 till January 2015 at the Clinical Oncology Department, Faculty of Medicine, Ain Shams University was $59 \%$ of cases within the first three months of completing chemotherapy and about $83 \%$ of them had persistent neuropathy for more than six months after last cycle of paclitaxel.

Data of the current trial is comparable to that reported by Hershman et al who stated that patients with breast cancer who received taxane-based adjuvant chemotherapy had neuropathy symptoms up to 2 years after completing treatment ${ }^{22}$. The incidence of all grades of paclitaxel induced neuropathy range from $57-83 \%$ and with severity in $2-33 \%$ of patients ${ }^{23}$.

The published guidelines by the American Society of Clinical Oncology for the prevention of chemotherapy-induced peripheral neuropathy relied on 42 randomized controlled trials wrapped in a systematic review that investigated 18 different agents ${ }^{24}$. The review showed that no agent has an obvious role in the prevention of chemotherapy-induced peripheral neuropathy. Moreover, 16 out of the 42 studies had a sample size of less than 50 patients.

The rationale for this study is based on the role of NGF in developing the dorsal route ganglion and 
Table 1: Baseline patients' characteristics

\begin{tabular}{llllll}
\hline & Test & & Control & \multicolumn{2}{c}{ p value } \\
\cline { 2 - 6 } & Mean $\mathbf{\pm}$ SD & Median $(\min -\mathbf{m a x})$ & Mean \pm SD & Median $(\min -\mathbf{m a x})$ & 0.738 \\
\hline Age $($ years) & $50.3 \pm 11.2$ & $48.5(31-70)$ & $51.6 \pm 10.8$ & $50.5(33-70)$ & 1 \\
\hline Paclitaxel dose $\left(\mathbf{m g} / \mathbf{m}^{\mathbf{2}}\right)$ & $143.5 \pm 13$ & $159(135-170)$ & $141.5 \pm 13$ & $148(135-160)$ & 1 \\
\hline Body surface area & $1.8 \pm 0.2$ & $1.8(1.4-2)$ & $1.8 \pm 0.2$ & $1.8(1.4-2)$ & 1 \\
\hline
\end{tabular}

Table 2: EORTC QLQ-CIPN20 neuroscores and nerve growth factor levels in both groups

\begin{tabular}{|c|c|c|c|c|c|}
\hline & \multicolumn{2}{|l|}{ Test } & \multicolumn{2}{|l|}{ Control } & \multirow[t]{2}{*}{ p value * } \\
\hline & Mean \pm SD & Median (min - max) & Mean \pm SD & Median (min - max) & \\
\hline \multicolumn{6}{|c|}{ EORTC QLQ-CIPN20 } \\
\hline Baseline (day 1) & $18 \pm 0$ & $18(18-18)$ & $18 \pm 0$ & $18(18-18)$ & \\
\hline $1^{\text {st }}$ cycle (day 21$)$ & $19.5 \pm 0.5$ & $19(19-20)$ & $28.9 \pm 7.8$ & $32(18-42)$ & $<0.001$ \\
\hline $2^{\text {nd }}$ cycle (day 42) & $18.8 \pm 0.8$ & $19(18-20)$ & $33.7 \pm 7.4$ & $32(22-44)$ & $<0.001$ \\
\hline $3^{\text {rd }}$ cycle (day 63) & $18.2 \pm 0.4$ & $18(18-19)$ & $37.3 \pm 5.5$ & $39(28-44)$ & $<0.001$ \\
\hline $\mathrm{p}$ value $* *$ & $<0.001$ & & $<0.001$ & & \\
\hline \multicolumn{6}{|c|}{ Nerve growth factor $(\mathrm{ng} / \mathrm{ml})$} \\
\hline Baseline & $23.3 \pm 31.3$ & $7.8(0.4-92.2)$ & $13.6 \pm 9.8$ & $9.5(3.3-35)$ & 1 \\
\hline End & $387.8 \pm 486.4$ & $255.4(188.6-2300.5)$ & $3.8 \pm 4.3$ & $1.7(1-16)$ & $<0.001$ \\
\hline $\mathrm{p}$ value $* *$ & $<0.001$ & & $<0.001$ & & \\
\hline Change & 364.5 & 247.6 & -9.8 & -7.8 & $<0.001$ \\
\hline
\end{tabular}

* Between groups; ** Within the same group over time

Table 3: Myalgia and arthralgia in both groups using NCI -CTCAE 4.0.

\begin{tabular}{lllll}
\hline Myalgia and arthralgia & Test & \multicolumn{2}{c}{ Control } & p value * \\
\cline { 2 - 4 } & Mean $\mathbf{E D}$ & Median & Mean $\mathbf{E D}$ & Median \\
\hline $\mathbf{1}^{\text {st }}$ cycle & $1.05 \pm 1$ & 1 & $1.1 \pm 0.8$ & 1 \\
\hline $\mathbf{2}^{\text {nd }}$ cycle & $2.1 \pm 0.7$ & 2 & $1.9 \pm 1$ & 2 \\
\hline $\mathbf{3}^{\text {rd }}$ cycle & $2.7 \pm 0.7$ & 3 & $2.9 \pm 0.7$ & 3 \\
\hline p value $* *$ & 0.042 & & 0.042 & 1 \\
\hline
\end{tabular}

* Between groups; ** Comparison was done between first, second and third cycle; NCI-CTCAE 4.0: National Cancer Institute Common Terminology Criteria for Adverse Event version 4.

Table 4: Comparing the change in laboratory parameters between the two groups

\begin{tabular}{llll}
\hline Laboratory parameter & Test & Control & p value \\
\cline { 2 - 4 } & Mean change \pm SD & Mean change \pm SD & \\
\hline Hemoglobin & $1.3 \pm 1.2$ & $1 . .2 \pm 1.1$ & 0.098 \\
\hline Uric acid & $0.3 \pm 0.2$ & $-0 . .5 \pm 0.3$ & 1 \\
\hline Blood Urea Nitrogen & $1.2 \pm 13.5$ & $-1.2 \pm 2.2$ & 0.994 \\
\hline Creatinine & $-0.3 \pm 0.2$ & $-0.5 \pm 0.9$ & 1 \\
\hline Alanine transaminase & $-1.8 \pm 2.8$ & $-1.3 \pm 1.9$ & 1 \\
\hline Aspartate transaminase & $-2.7 \pm 5.3$ & $-1.4 \pm 0.9$ & 1 \\
\hline Total bilirubin. & $-0.6 \pm 0.3$ & $-0.8 \pm 0.3$ & 1 \\
\hline
\end{tabular}

maintaining its functions ${ }^{25}$. Moreover, NGF can rescue neuronal cells from apoptosis 26,27 . The potentiality of NGF in protecting the neurons was illustrated in several studies performed in vitro on the neurotoxicity of paclitaxel, cisplatin and suramine ${ }^{28,29}$. Nerve growth factor prevented the decrease in compound motor action potential and increased the threshold of thermally induced pain induced by cisplatin and paclitaxel in animal models ${ }^{30}$.

Even though NGF has not been tested in humans; it demonstrated a counteracting effect for the reduction of neurite outgrowth from rat dorsal root ganglion in vitro, induced by cisplatin, vincristine or paclitaxel ${ }^{31}$. Hence, the research team of this pilot study hypothesized that exogenous NGF may have a potential role in preventing PIPN.

The main mechanism of action of cerebrolysin is to help brain neurons function more efficiently and more in sync with each other. Cerebrolysin has a neutrophic activity and neuroprotective properties that do not only prevent degeneration of neurons in the central nervous system, but it also stimulates the growth of new neurons.

Since there was no previous experience in preventing human PIPN with cerebrolysin and the absence of clear dose schedules, the authors of the current study used the cerebrolysin dose recommended in treating patients with cerebrovascular stroke ${ }^{32}$.

The current study revealed that the QLQ-CIPN20 scores remained essentially flat across time points in the test group, which supports the conclusion that the exogenous NGF administration may have prevented PIPN. On the other hand, the control group experienced significant deterioration in their QLQ-CIPN20 scores by the $3^{\text {rd }}$ cycle compared to the $1^{\text {st }}$. These findings indicate a possible beneficial effect of exogenous NGF administration on nerves due to its neuroprotective and 
neurotropic functions that halted the anticipated PIPN occurrence.

The data of the current study agrees with prior phase II trials of NGF. Patients with diabetic and HIV polyneuropathy were subjected to two placebocontrolled randomized trials that used recombinent human NGF (rh-NGF). Both trials included 250 and 270 patients respectively; rh-NGF was used in a dose range 0.1 or $0.3 \mu \mathrm{g} / \mathrm{kg}$ subcutaneously, thrice weekly and twice weekly for 6 months and 18 weeks respectively. The efficacy results of these studies were encouraging in terms of improvement of neuropathic pain ${ }^{33-35}$. On the contrary, rh-NGF was not effective in a phase III trial done by Apfel et al. They enrolled 1019 diabetic patients and treated them with rhNGF 3 times a week for 48 weeks by subcutaneous injection. The negative result of this study may be explained by the low dosage of NGF, limited by the occurrence of toxicities. Other causes may be considered, as the eligibility criteria, end points characterization, methods of neuropathy measurement and may be the low quality of rhNGF used ${ }^{36}$.

The current study showed a clear difference in the serum levels of NGF between the study groups. The final human NGF levels were significantly lower in the control group compared to their initial baseline values, with a corresponding deterioration in their neuroscore. While, the test group NGF levels at the $3^{\text {rd }}$ cycle were significantly higher than their base line levels, with a corresponding stability of their neuroscores. Moreover, there was a significantly positive correlation between the neuroscore and the NGF levels. It can be suggested from these findings that there is a correlation between the circulating level of endogenous NGF and the occurrence of PIPN. However, properly-designed studies will be needed to determine if a decrease in circulating NGF is associated with worsening PIPN and to confirm that increasing NGF levels is protective. These results are consistent with the data reported by Cavaletti et al who stated that there is an obvious inverse correlation between the decrease in NGF level and the severity of CIPN ( $r=-0.579 ; \mathrm{p}<0.001)^{37}$.

Safety issues in the current trial relied on the phase I trial of rh-NGF in healthy subjects that reported tolerable side effects, such as muscle pain and boring skin sensation at the site of injection ${ }^{38,39}$. The expected efficacy issues of the current work relied on a phase II trial performed on 250 diabetic patients with neuropathy. This trial showed a marked improvement in neuropathy symptoms in the NGF-treated group. However, this was at the cost of increased toxicity such as muscle and joint pains 33,34 . In this pilot, there was no significant difference between both groups in all evaluated laboratory parameters. Moreover, no difference was observed between groups in the incidence of myalgia and arthralgia. These findings indicate a favorable safety profile of cerebrolysin and lack of potential harmful toxicities accompanying its administration.

The difference between the toxicity data of the current study and that of previous studies by Apfel et al in terms of myalgia, arthralgia and injection site effects may be attributed to the difference between the chemical structure and route of NGF administration ${ }^{33,34}$. Apfel used rh-NGF subcutaneously, while the current study used procaine peptide with NGF properties intramuscularly that is already being used in treating some diseases such as cerebral stroke. Moreover, both groups in the current study received paclitaxel which normally causes myalgia and arthralgia. Data reported by Ziganshina et al who evaluated the use of cerebrolysin for acute ishaemic stroke stated that cerebrolysin did not demonstrated serious adverse reactions ${ }^{40}$.

Finally, the current study demonstrated acceptable recruitment rate, refusal rate and non-compliance rate. The eligibility and exclusion criteria were applicable, and there were no time and budget problems (cerebrolysin was bought by the authors upon their own expense) and we did not encounter potential human and data optimization problems.

In conclusion, exogenous NGF demonstrated a potential neuroprotective effect against PIPN in breast cancer patient as evidenced by the stabilization of neuroscores at enrollment versus the neuroscores after the 3 paclitaxel cycles. Moreover, this pilot demonstrated the potential inverse correlation between the serum levels of NGF and the severity of PIPN.

The data presented makes a sensible recommendation for performing the study as a properlydesigned prospective combined Phase I/II randomized controlled trial with cross-over for properly testing the neuroprotective effect of exogenous NGF in breast cancer patients receiving paclitaxel.

\section{Conflict of Interest}

The authors declare that they have no conflict of interest.

\section{REFERENCES}

1. Cavaletti G, Marmiroli P. Chemotherapy induced peripheral neurotoxicity. Nat Rev Neurol. 2010; 6(12): 657-666.

2. Bookman MA, McGuire WP 3rd, Kilpatrick D, et al. Carboplatin and paclitaxel in ovarian carcinoma: a phase I study of the Gynecologic Oncology Group. J Clin Oncol. 1996; 14(6): 1895-1902.

3. Gordon AN, Stringer CA, Matthews CM, Willis DL, Nemunaitis J. Phase I dose escalation of paclitaxel in patients with advanced ovarian cancer receiving cisplatin: rapid development of neurotoxicity is doselimiting. J Clin Oncol. 1997; 15(5): 1965-1973.

4. Cavaletti G, Bogliun G, Marzorati L, et al. Peripheral neurotoxicity of taxol in patients previously treated with cisplatin. Cancer. 1995; 75(5): 1141-1150.

5. Swain SM, Arezzo JC. Neuropathy associated with microtubule inhibitors: diagnosis, incidence, and management. Clin Adv Hematol Oncol. 2008; 6(6): 445-467.

6. Hershman DL, Weimer LH, Wang A, et al Association between patient reported outcomes and quantitative sensory tests for measuring long-term neurotoxicity in breast cancer survivors treated with adjuvant paclitaxel chemotherapy. Breast Cancer Res Treat. 2011; 125(3): 767-774.

7. Lee JJ, Swain SM. Peripheral neuropathy induced by microtubule stabilizing agents. J Clin Oncol. 2006; 24(10): 1633-1642. 
8. Pachman DR, Barton DL, Waston JC, Loprinzi CL. Chemotherapy induced peripheral neuropathy prevention and treatment. Clin Pharmacol Ther. 2011; 90: 377-387.

9. Kaczor T. The therapeutic effects of acetyl-L-carnitine on peripheral neuropathy. Nat Med J. 2010; 2(8): 7-11.

10. Xiao WH, Bennett GJ. Chemotherapy-evoked neuropathic pain: Abnormal spontaneous discharge in A-fiber and C-fiber primary afferent neurons and its suppression by acetyl-L-carnitine. Pain. 2008; 135(3): 262-270.

11. Pittenger G, Vinik A. Nerve growth factor and diabetic neuropathy. Exp Diabesity Res. 2003; 4(4): 271-285.

12. Anand P, Rudge P, Mathias CJ, et al. New autonomic and sensory neuropathy with loss of adrenergic sympathetic function and sensory neuropeptides. Lancet. 1991; 337(8752): 1253-1254.

13. Anand P, Terenghi G, Warner G, Kopelman P, Williams-Chestnut RE, Sinicropi DV. The role of endogenous nerve growth factor in human diabetic neuropathy. Nat Med. 1996; 2(6):703-707.

14. De Santis S, Pace A, Bove L, et al. Patients treated with antitumor drugs displaying neurological deficits are characterized by a low circulating level of nerve growth factor. Clin Cancer Res. 2000; 6(1): 90-95.

15. Miltenburg NC, Boogend W. Chemotherapy induced neuropathy: A comprehensive study. Cancer Treat Rev. 2014; 40(7): 872-882.

16. US Department of Health and Human Services. Common Terminology Criteria for Adverse Events. (CTCAE). Version 4.0 Published May 28, (2009). http.//www.hrc.govt.nz/sites/default/files/CTCAE\%20 mannula\%20\%20 DMCC.pdf. Accessed December 2014.

17. Postma TJ, Aronson NK, Heimana JJ, et al. The development of an EORTC quality of life questionnaire to assess chemotherapy induced peripheral neuropathy: the QLQ-CIPN20. Eur J Cancer. 2005; 41(8): 1135-1139.

18. Lavoie Smith EM, Barton DL, Qin R, Steen PD, Aaronson NK, Loprinzi CL. Assessing patient reported peripheral neuropathy; The reliability and validity of the European Organization for Research and Treatment of Cancer. QLQ-CIPN20 questionnaire. Qual Life Res. (2013); :(22)10:2787-2799.

19. Gauthier S, Proaño JV, Jia J, Froelich L, Vester JC, Doppler E. Cerebrolysin in mild-to-moderate alzheimer's disease: a meta-analysis of randomized controlled clinical trials. Dement Geriatr Cogn Disord. 2015; 39(5-6): 332-347.

20. Plosker GL, Gauthier S. Cerebrolysin: a review of its use in dementia. Drugs Aging. 2009; 26(11): 893-915.

21. www.everpharma.com/products/cerebrolysinr/ Accessed: 31 December 2016.

22. Hershman DL, Weimer LH, Wang A, et al. Association between patients reported outcomes and quantitative sensory tests for measuring long-term neurotoxicity in breast cancer survivors treated with adjuvant paclitaxel chemotherapy. Breast Cancer Res Treat. 2011; 125(3): 767-774.

23. Rivera E, Cianfrocca M. Overview of neuropathy associated with taxanes for the treatment of metastatic breast cancer. Cancer Chemother Pharmacol. 2015; 75(4): 659-670.

24. Hershmann DL, Lacchetti C, Dworkin RH, et al. Prevention and management of chemotherapy induced peripheral neuropathy in survivors of adult cancers:
American Society of Clinical Oncology clinical practice guideline. J Clin Oncol. 2014; 32(18): 19411967.

25. Apfel SC, Arezzo JC, Lipson L, Kessler JA. Nerve growth factor prevents experimental cisplatin neuropathy. Ann Neurol. 1992; 31(1): 76-80.

26. Jensen LM, Zhang Y, Shooter EM. Steady-state polypeptide modulations associated with nerve growth factor (NGF)-induced terminal differentiation and NGF deprivation-induced apoptosis in human neuroblastoma cells. J Biol Chem. 1992; 267(27): 19325-19333.

27. Rabizadeh S, Oh J, Zhong LT, et al. Induction of apoptosis by the low-affinity NGF receptor. Science. 1993; 261(5119): 345-348.

28. Peterson ER, Crain SM. Nerve growth factor attenuates neurotoxic effects of taxol on spinal cordganglion explants from the fetal mice. Science. 1982; 217(4557): 377-379.

29. Hayakawa K, Sobue G, Itoh T, Mitsuma T. Nerve growth factor prevents neurotoxic effects of cisplatin, vincristine and taxol on adult rat sympathetic ganglion explants in vitro. Life Sci. 1994; 55(7): 519525.

30. Apfel SC, Lipton RB, Arezzo JC, Kessler JA. Nerve growth factor prevents toxic neuropathy in mice. Ann Neurol. 1991; 29(1): 87-90.

31. Konings PN, Makkink WK, van Delft AM, Ruigt GS. Reversal by NGF of cytostatic drug-induced reduction of neurite outgrowth in rat dorsal root ganglia in vitro. Brain Res. 1994; 640(1-2): 195-204.

32. https://www.drugs.com/international/cerebrolysin.html . Accessed 31 December (2016).

33. Apfel SC, Kessler JA, Adornato BT, Litchy WJ, Sanders C, Rask CA. Recombinant human nerve growth factor in the treatment of diabetic polyneuropathy. NGF Study Group. Neurology. 1998; 51(3): 695-702.

34. Apfel SC. Nerve growth factor for the treatment of diabetic neuropathy: what went wrong, what went right, and what does the future hold? Int Rev Neurobiol. 2002; 50:393-413.

35. McArthur JC, Yiannoutsos C, Simpson DM, et al. A phase II trial of nerve growth factor for sensory neuropathy associated with HIV infection. AIDS Clinical Trials Group Team 291. Neurology. 2000; 54(5):1080-1088.

36. Apfel SC, Schwartz S, Adornato BT, et al. Efficacy and safety of recombinant human nerve growth factor in patients with diabetic polyneuropathy: a randomized controlled trial. rhNGF Clinical Investigator Group. JAMA. 2000; 284(17): 2215 2221.

37. Cavaletti G, Bogliun G, Marzorati L, et al. Early predictors of peripheral neurotoxicity in cisplatin and paclitaxel combination chemotherapy. Ann Oncol. 2004; 15(9): 1439-1442.

38. Rogers BC. Development of recombinant human nerve growth factor (rhNGF) as a treatment for peripheral neuropathic disease. Neurotoxicology. 1996; 17(3-4): 865-870.

39. Petty BG, Cornblath DR, Adornato BT, et al. The effect of systemically administered recombinant human nerve growth factor in healthy human subjects. Ann Neurol. 1994; 36(2): 244-246.

40. Ziganshina LE, Abakumova T, Vernay L. Cerebrolysin for acute ischaemic stroke. Stroke. 2017; 48(9): e245e246. 